\title{
An Ordering for Groups of Pure Braids and Fibre-type Hyperplane Arrangements
}

\author{
Djun Maximilian Kim and Dale Rolfsen*
}

April 11, 2002

\section{Introduction and Statement of Results}

This paper concerns the Artin braid groups $B_{n}$ and especially the subgroups $P_{n}$ of pure braids. Our main result is the explicit construction of a strict total ordering, $<$, of pure braids which is invariant under multiplication on both sides (for $\alpha, \beta, \gamma \in P_{n}, \alpha<\beta$ implies $\alpha \gamma<\beta \gamma$ and $\gamma \alpha<\gamma \beta$ ) and has the following properties:

1. There is a straightforward algorithm to decide which of two given pure braids is the greater. It is, in a sense, an extension of Artin's solution of the word problem for $P_{n}$.

2. The ordering respects the standard inclusions $P_{n} \subset P_{n+1}$, and so defines an ordering on the direct limit $P_{\infty}$. It is also compatible with the homomorphism $f: P_{n+1} \rightarrow P_{n}$ which "forgets the last string," meaning that $\alpha<\beta$ implies $f(\alpha) \leq f(\beta)$.

3. The pure braids $\beta \in P_{n}$ which are positive in the sense of Garside [14] are also positive in our ordering. That is, if $\beta$ is a nontrivial pure braid expressible in the standard braid generators $\sigma_{i}$ with no negative exponents $\left(\beta \in P_{n}^{+}=P_{n} \cap B_{n}^{+}\right)$then $1<\beta$, where 1 denotes the identity $n$-string braid. (see sec. 8 )

4. $P_{n}^{+}$is well-ordered under our ordering.

5. Our ordering of $P_{n}$ extends Garside's partial ordering of $P_{n}^{+}$. (see sec. 9)

The ordering is defined by a combination of the "combing" technique of Artin and the Magnus expansion of free groups in rings of formal power series. The existence of an ordering of $P_{n}$, invariant under multiplication on both

* This research has been partially supported by a grant from the Canadian Natural Sciences and Engineering Research Council. The second author wishes to acknowledge the hospitality of the University of Bourgogne and the Mathematics Institute of the University of Geneva during part of this work. 
sides, implies that the group ring $\mathbf{Z} P_{n}$ embeds in a division ring, using a result proved independently by Malćev [19] and Neumann $[22]^{1}$. An important result of Dehornoy [8] (see also [13] and [29]) is that the full braid groups $B_{n}$ can be given a strict total ordering which is invariant under multiplication on one side (but not both). We will see that Dehornoy's ordering of $B_{n}$ and our ordering of $P_{n}$ are fundamentally incompatible; indeed any right- or left-ordering of $B_{n}$ is necessarily incompatible with any bi-ordering of $P_{n}$, according to [27].

In the following sections, we describe a few basics of the braid groups, orderable groups, Artin's combing, Magnus expansions, Dehornoy's ordering and establish our particular conventions. We also discuss the connection with partial orderings which have been defined by Garside and Elrifai-Morton.

We are grateful to L. Paris for pointing out that our methods also apply to a large class of complex hyperplane arrangements, those of fibre type, as defined in [12]. Thus we can define explicit orderings to show the following:

THEOREM: The fundamental group of the complement of a complex hyperplane arrangement of fibre type can be given a strict total ordering which is invariant under multiplication on both sides.

As pointed out in [28], the existence of a bi-ordering of $P_{n}$ (as well as the fibre-type hyperplane arrangement groups) follows directly from the observation of [12] that these groups are residually nilpotent, torsion-free (using the main result of [23]). The contribution of the present paper is the explicit construction with the properties cited above, perhaps most notably the well-ordering of Garside-positive pure braids.

\section{Braid groups}

Emil Artin [1] defined the braid groups $B_{n}$ as isotopy classes of $n$ disjoint strings embedded in 3-space, monotone in a given direction, and beginning and ending at certain specified points. The product of two braids is given by concatenation. These groups have played an extremely important rôle in topology, analysis, algebra and theoretical physics. Artin showed that $B_{n}$ admits a presentation with generators $\sigma_{1}, \ldots, \sigma_{n-1}$, and relations

$$
\begin{array}{rlrl}
\sigma_{i} \sigma_{j} & =\sigma_{j} \sigma_{i} & \text { if }|i-j| & >1, \quad 1 \leq i, j \leq n-1 \\
\sigma_{i} \sigma_{i+1} \sigma_{i} & =\sigma_{i+1} \sigma_{i} \sigma_{i+1} & i & =1, \ldots, n-2,
\end{array}
$$

where the generators $\sigma_{i}$ can be interpreted geometrically as the so-called elementary braids (shown in figure 2) whose only crossing takes the $(i+1)$-st strand over the $i$-th; this corresponds to a right-hand screw twist.

Corresponding to any braid is a permutation of the set $\{1, \cdots, n\}$ recording how the strings connect the endpoints. In particular, a braid whose permutation is the identity is called a pure braid. These form a normal subgroup $P_{n}$ of

\footnotetext{
${ }^{1}$ Added in proof: P. Linnell has announced that $\mathbf{Z} B_{n}$ also embeds in a division ring.
} 


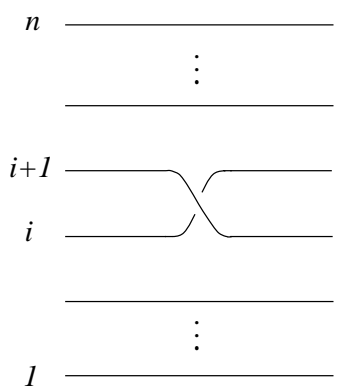

Figure 1: The elementary braid $\sigma_{i}$

index $n$ ! in $B_{n}$, and fit into an exact sequence

$$
1 \rightarrow P_{n} \rightarrow B_{n} \rightarrow S_{n} \rightarrow 1 .
$$

\section{Automorphisms of free groups}

Let $F_{n}$ be a free group of rank $n$, with generators $x_{1}, \ldots, x_{n}$. Artin showed that $B_{n}$ embeds in $\operatorname{Aut}\left(F_{n}\right)$, the automorphism group of the free group, in the following way. Let $\mathbf{D}_{n}:=D^{2}-Q_{n}$ denote the disk with $n$ punctures $Q_{n}=\left\{p_{1}, \ldots, p_{n}\right\}$ arranged in order along a straight line in $D^{2} . F_{n}$ can be identified with $\pi_{1}\left(\mathbf{D}_{n}\right)$. A braid can be regarded as the graph of a motion of $n$ distinct points in the disk, beginning and ending in $Q_{n}$; this motion extends to the whole disk, so that a braid gives rise to a self-homeomorphism of $\mathbf{D}_{n}$, unique up to isotopy, fixing the punctures and boundary of $D^{2}$. Passing to the fundamental group, this induces an automorphism of $F_{n}$.

To be precise, let $x_{1}, \ldots, x_{n}$ be loops in $\mathbf{D}_{n}$ representing generators of $\pi_{1}\left(\mathbf{D}_{n}\right)$, oriented in the clockwise sense, as shown in Figure 2. The arcs stand for loops which are small regular neighbourhoods of the arcs.

The braid $\sigma_{i}$ corresponds to the (say right-hand) screw motion interchanging $p_{i}$ and $p_{i+1}$ and fixed off a small neighborhood of the interval between these points. The automorphism corresponding to $\sigma_{i}$ is given by

$$
\begin{aligned}
x_{i} & \mapsto x_{i} x_{i+1} x_{i}{ }^{-1}, \\
x_{i+1} & \mapsto x_{i}, \\
x_{j} & \mapsto x_{j} \quad(j \neq i, i+1 .)
\end{aligned}
$$

This action is illustrated in Figure 2. Thus a braid $\beta$ will take $x_{i}$ to $w_{i} x_{\pi(i)} w_{i}{ }^{-1}$, where $\pi$ is the permutation corresponding to $\beta$, and $w_{i}$ also depends on $\beta$. It is well-known that this determines an embedding of $B_{n}$ into $\operatorname{Aut}\left(F_{n}\right)$. We point out for later reference that a pure braid corresponds to an automorphism which is a "local conjugation," i.e., one in which each generator is sent to some conjugate of itself.

Further details may be found, for example, in [5], or in [7]. 

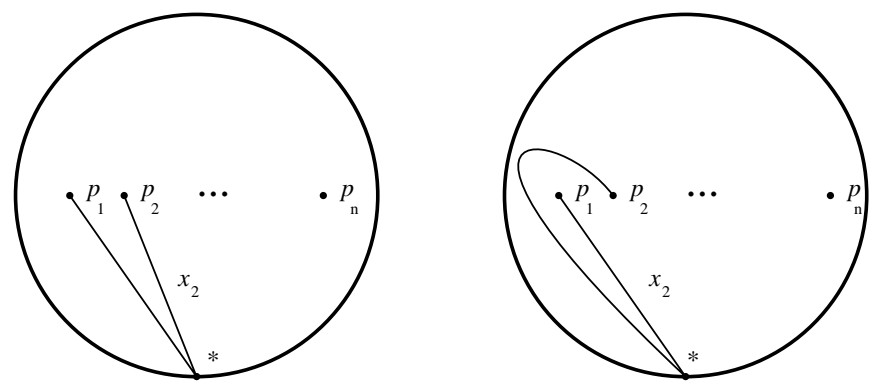

Figure 2: The action of $\sigma_{1}$ on $\pi_{1}\left(\mathbf{D}_{n}\right)$

\section{Ordered groups}

If $G$ is a group whose elements can be given a strict total ordering < which is right-invariant,

$$
g<h \Rightarrow g k<h k
$$

for all $g, h, k \in G$, then $(G,<)$ is said to be a right-ordered group. If the ordering is also left-invariant, we say $(G,<)$ is a bi-ordered group (also called fully-ordered, or simply "ordered" in the literature.)

A right-orderable group $G$ can be recognized by the existence of a "positive cone" $\mathcal{P} \subset G$. A group $G$ is right-orderable if and only if there exists $\mathcal{P} \subset G$ such that: (1) $\mathcal{P}$ is multiplicatively closed: $\mathcal{P} \cdot \mathcal{P} \subset \mathcal{P}$, and (2) $G \backslash\{1\}=\mathcal{P} \amalg \mathcal{P}^{-1}$ where $\mathcal{P}^{-1}=\left\{p^{-1} \mid p \in \mathcal{P}\right\}$, i.e., $\mathcal{P}$ partitions $G$. For if $G$ is a right orderable group, take $\mathcal{P}=\{g \mid 1<g\}$. Conversely, if such a subset $\mathcal{P}$ exists, we may define a right order on $G$ by $g<h \Leftrightarrow h g^{-1} \in \mathcal{P}$. (Note: the criterion $g^{-1} h \in \mathcal{P}$ would give a left-invariant ordering; a group is right-orderable if and only if it is left-orderable, but the orderings may differ.)

$G$ is bi-orderable if and only if properties (1) and (2) above hold, for some subset $\mathcal{P}$ and in addition, (3): for all $g \in G, g \mathcal{P} g^{-1} \subset \mathcal{P}$ (normality).

It is clear that subgroups of right-orderable (resp. bi-orderable) groups are right-orderable (resp. bi-orderable) groups. The theory of orderable groups is over a century old. A classical theorem of Hölder [15] asserts that if an orderable group is Archimedean, then it is isomorphic (algebraically and in the ordering sense) with a subgroup of the additive reals, and therefore abelian. An ordering on a group is said to be Archimedean if whenever $1<x<y$ in the group, there exists an integer $p$ such that $y<x^{p}$.

Orderability is a strong property. For example, if $G$ is right-orderable, then $G$ is torsion-free. Furthermore, if $R$ is a ring with no zero-divisors, and $G$ is right-orderable, then the group ring $R G$ has no zero-divisors and no units, other than the "trivial units" of the form $r g$ where $r$ is a unit in $R$ and $g \in G$. It is nontrivial, but well-known (see [23], [4], [9], [16]) that free groups are biorderable (we will present a proof shortly). More generally, Vinogradov [30] proved that bi-orderability is preserved under free products. Good references on ordered groups are [26], [21], or [17]. 
Right orderability is easily seen to be preserved under extensions, that is, if $1 \rightarrow G \rightarrow K \rightarrow H \rightarrow 1$ is an exact sequence of groups and $G$ and $H$ are right orderable, so is $K$, by taking $k \in K$ to be positive iff its image in $H$ is positive, or the image is the identity and $k$ is positive in $G$. However, bi-orderability is not quite so well-behaved, being preserved under direct products, but not necessarily even semi-direct products. If $G$ and $H$ are bi-orderable, then $G \times H$ is also biorderable: simply take the lexicographic ordering: $(g, h)<\left(g^{\prime}, h^{\prime}\right) \Longleftrightarrow g<_{G} g^{\prime}$ or $g=g^{\prime}$ and $h<_{H} h^{\prime}$.

Now suppose $G$ acts on $H$, the action being denoted by $h \mapsto h^{g}$. In this situation, we can define the semi-direct product $G \ltimes H$ to have underlying set $G \times H$, but taking as the product $(g, h)\left(g^{\prime}, h^{\prime}\right)=\left(g g^{\prime}, h^{g^{\prime}} h^{\prime}\right)$. The Klein bottle group

$$
\left\langle x, y \mid y x y^{-1}=x^{-1}\right\rangle
$$

is not bi-orderable, although it is a semi-direct product of two infinite cyclic groups (which are, of course, bi-orderable). The reason this group cannot be bi-orderable is that $x$ and $x^{-1}$ are conjugate, and so one is positive if and only if the other one is positive, which leads to a contradiction. A similar argument can be made in the braid group $B_{3}$, taking $x=\sigma_{1} \sigma_{2}^{-1}$ and $y=\sigma_{1} \sigma_{2} \sigma_{1}$. It follows that none of the braid groups $B_{n}, n \geq 3$ are bi-orderable, as observed in [24].

We shall need the following lemma, whose proof is routine.

Lemma 1 Let $G$ and $H$ be bi-ordered groups. Then the lexicographical order on $G \ltimes H$ is a bi-ordering if and only if the action of $G$ on $H$ preserves the order on $H$ (equivalently, $\left(\mathcal{P}_{H}\right)^{g} \subset \mathcal{P}_{H}$ for all $g \in G$.)

\section{The Magnus ordering of the free group $F_{n}$}

Let $F_{n}$ denote the free group of rank $n$, generated by $x_{1}, \ldots, x_{n}$. By using a representation of $F_{n}$ into a sufficiently large ring, due to Magnus [20], we can produce a bi-ordering with special invariance properties.

Define $\mathbf{Z}\left[\left[X_{1}, X_{2}, \ldots, X_{n}\right]\right]$ to be the ring of formal power series in the noncommuting variables $X_{1}, \ldots, X_{n}$, and let $\mu: F_{n} \rightarrow \mathbf{Z}\left[\left[X_{1}, X_{2}, \ldots, X_{n}\right]\right]$ be the Magnus map, defined on generators by

$$
\mu: \begin{cases}x_{i} & \mapsto 1+X_{i} \\ x_{i}{ }^{-1} & \mapsto 1-X_{i}+X_{i}{ }^{2}-X_{i}^{3}+\cdots\end{cases}
$$

Each term of a formal series has a degree, simply the sum of the exponents, and we use the usual notation $\mathcal{O}(d)$ to denote the sum of all terms of degree at least $d$. As noted in [20], the subset $\mathcal{G}=\{1+\mathcal{O}(1)\}$ of $\mathbf{Z}\left[\left[X_{1}, X_{2}, \ldots, X_{n}\right]\right]$ is a subgroup. The inverse of an element $1+W$ is simply $1-W+W^{2}-\cdots$, even when $W \in \mathcal{O}(1)$ is itself an infinite series. Also shown in [20] is the following.

Theorem 2 (Magnus) The map $\mu: F_{n} \rightarrow \mathcal{G}$ is injective. 
Because of this, we may omit mention of the map $\mu$ and identify $F_{n}$ with its image, writing $x_{1}=1+X_{1}$, etc.

Definition 3 We now define an ordering on $\mathbf{Z}\left[\left[X_{1}, X_{2}, \ldots, X_{n}\right]\right]$. See [20], chapter 5, exercise 5.6.10, and also [3]. First declare $X_{1} \prec X_{2} \prec \cdots \prec X_{n}$. If $V, W$ are two distinct series, consider the smallest degree at which they differ, and sort the monomial terms of that degree with variables in lexicographic order, using $\prec$. Compare the coefficients of the first term, when written in the order described, at which $V$ and $W$ differ. Declare $V<W$ precisely when the coefficient of that term in $V$ is smaller than the corresponding coefficient in $W$. We call this the Magnus ordering on $\mathbf{Z}\left[\left[X_{1}, X_{2}, \ldots, X_{n}\right]\right]$.

Example 1 Under the Magnus map, we have $x_{1}=1+X_{1}$ and $x_{2}=1+X_{2}$. The images differ in that $x_{1}$ has coefficient 1 at term $X_{1}$ while $x_{2}$ has coefficient 0 in this term. Hence $x_{1}>x_{2}$. Similarly, we have $x_{1}>x_{2}>\cdots>x_{n}>1$.

Theorem 4 The Magnus order on $\mathbf{Z}\left[\left[X_{1}, X_{2}, \ldots, X_{n}\right]\right]$ induces a bi-ordering on $\mathcal{G}$ and hence on $F_{n}$. Moreover, this ordering of $F_{n}$ is preserved under any $\varphi \in \operatorname{Aut}\left(F_{n}\right)$ which induces the identity on $H_{1}\left(F_{n}\right)=F_{n} /\left[F_{n}, F_{n}\right]$.

Proof. The ordering is clearly invariant under addition, but not necessarily under multiplication in general (for example multiplication by -1 reverses order.) However, we shall establish right-invariance under multiplication in $\mathcal{G}$ by verifying that if $u, v \in \mathcal{G}$ then we have

$$
u<v \Leftrightarrow v u^{-1}>1 \text {. }
$$

Write $u=1+U$ and $v=1+V$ where $U, V \in \mathcal{O}(1)$. It is clear from the definition that $u<v$ if and only if $V-U>0$. In the calculation

$$
v u^{-1}=(1+V)\left(1-U+U^{2}-\cdots\right)=1+V-U+R
$$

every term of the remainder $R=(V-U)\left(-U+U^{2}-U^{3}+\cdots\right)$ has degree exceeding that of the lowest degree term of $V-U$, and so $V-U$ is positive if and only if $v u^{-1}>1$. A similar calculation shows that $u<v \Leftrightarrow u^{-1} v>1$, and therefore the ordering is also left-invariant.

To prove the second statement, consider an automorphism $\varphi: F_{n} \rightarrow F_{n}$ which induces the identity on first homology. This means that $\varphi\left(x_{i}\right) x_{i}^{-1}$ is in the commutator subgroup $\left[F_{n}, F_{n}\right]$. As observed in [20], the image of $\left[F_{n}, F_{n}\right]$ lies in the subgroup $\{1+\mathcal{O}(2)\}$ of $\mathcal{G}$. For convenience, write $x_{i}^{\prime}=\varphi\left(x_{i}\right)$. The corresponding Magnus expansion is

$$
x_{i}^{\prime}=1+X_{i}^{\prime}=(1+\mathcal{O}(2))\left(1+X_{i}\right)=1+X_{i}+\mathcal{O}(2)
$$

and therefore

$$
X_{i}^{\prime}=X_{i}+\mathcal{O}(2)
$$

Now if $w$ is a word in the free group $F_{n}$, its image under $\varphi$ has Magnus expansion obtained from that of $w$ by replacing each occurence of $X_{i}$ by $X_{i}+\mathcal{O}(2)$. It 
follows that the first non-zero non-constant terms of $w$ and its image under $\varphi$ are identical, and therefore $\varphi$ preserves the positive cone of $F_{n}$, which is equivalent to being order-preserving.

\section{Artin Combing and the Structure of $P_{n}$}

There is a "natural" inclusion of $P_{n-1} \hookrightarrow P_{n}$, which adds an $n$-th strand to an $(n-1)$-strand pure braid $\beta$, as illustrated in Figure 3.

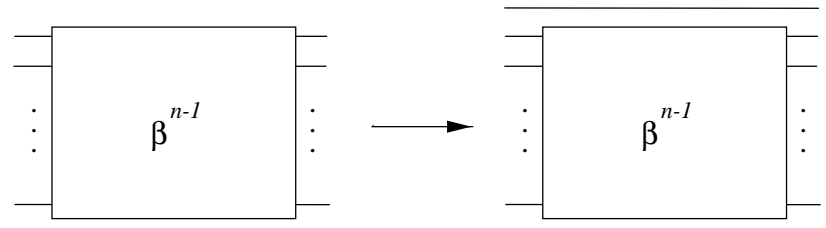

Figure 3: The natural inclusion of $P_{n-1} \hookrightarrow P_{n}$

There is also a retraction $f: P_{n} \rightarrow P_{n-1}$ defined by "deleting the $n$-th strand". Define $F_{n-1}$ to be the kernel of $f$; then $F_{n-1}$ consists of all braids with representatives for which strands $1,2, \ldots, n-1$ pass straight through and the $n$-th strand weaves among the remaining ones. This presents $F_{n-1} \cong$ $\pi_{1}\left(\mathbf{R}^{2} \backslash(n-1)\right.$ points $)$, a free group of rank $n-1$, as a normal subgroup of $P_{n}$. We may write $\beta \in P_{n}$ as $\beta=f(\beta) f(\beta)^{-1} \beta$. Note that $f(\beta) \in P_{n-1}$ while $f(\beta)^{-1} \beta \in F_{n-1}$.

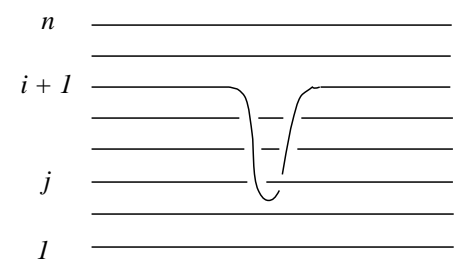

Figure 4: The generator $x_{j, i}$ of $F_{i}=\left\langle x_{1, i}, \ldots, x_{i, i}\right\rangle \subset P_{n}$.

For any $i \in\{1, \ldots, n-1\}$, we may thus consider the subgroup $F_{i} \subset P_{i} \subset P_{n}$ as consisting of all braids having all strands going straight across, except the one of index $i+1$, which may have crossings only with strings of lower index. Free generators $x_{1, i}, \ldots, x_{i, i}$ of $F_{i}$ are illustrated in Figure 4 . In terms of the Artin generators,

$$
x_{j, i}=\sigma_{i} \sigma_{i-1} \cdots \sigma_{j+1}{\sigma_{j}}^{2} \sigma_{j+1}{ }^{-1} \cdots \sigma_{i-1}{ }^{-1} \sigma_{i}{ }^{-1} .
$$

When the context of $F_{i}$ is understood, we will drop the second subscript and write simply $F_{i}=\left\langle x_{1}, \ldots, x_{i}\right\rangle$. 
Theorem 5 (Artin [2]) The map $\beta \mapsto\left(f(\beta), f(\beta)^{-1} \beta\right)$ is an isomorphism $P_{n} \cong P_{n-1} \ltimes F_{n-1}$. The action of $P_{n-1}$ on $F_{n-1}=\left\langle x_{1}, \ldots, x_{n-1}\right\rangle$ induces the identity on the abelianization $H_{1}\left(F_{n-1}\right)=F_{n-1} /\left[F_{n-1}, F_{n-1}\right]$.

Proof. The semi-direct product decomposition is well-known and immediate from the observation that $F_{n-1}$ is normal and intersects $P_{n-1}$ in precisely the identity element. For a good explanation, see [7], which also explains that the action of $P_{n-1}$ on $F_{n-1}$ turns out to be the Artin action of $B_{n-1}$ as described in Section 3, restricted to the pure braids. As remarked earlier, each such automorphism is a local conjugation, and therefore the identity upon abelianization.

The full "Artin combing" of $P_{n}$ is the iterated semi-direct product decomposition $\left.P_{n} \cong\left(\cdots\left(F_{1} \ltimes F_{2}\right) \ltimes F_{3}\right) \ltimes \cdots \ltimes F_{n-1}\right)$. This provides a unique factorization of a pure braid $\beta$ as a product $\beta=\beta_{1} \beta_{2} \ldots \beta_{n-1}$ in which the pure braid $\beta_{i}$ belongs to $F_{i}$. Thus we introduce coordinates in the semi-direct product:

$$
\beta=\left(\beta_{1}, \beta_{2}, \ldots, \beta_{n-1}\right),
$$

in which $\beta_{i}$ may be expressed in terms of the generators $x_{1}, \ldots, x_{i}$ of $F_{i}$.

Theorem 6 The lexicographic order on $P_{n} \cong F_{1} \ltimes F_{2} \ltimes F_{3} \ltimes \ldots \ltimes F_{n-1}$, with terms in the free factors compared using the Magnus order, is a bi-ordering.

Proof. This is just a recursive application of Theorem 5, Theorem 4 and Lemma 1.

We will refer to this order on $P_{n}$ as the Artin-Magnus order.

\section{$7 \quad$ Examples and properties of the ordering}

We will begin with some examples in $P_{4}=\left(F_{1} \ltimes F_{2}\right) \ltimes F_{3}$. An element $\beta \in P_{4}$ will be written $\beta=\left(\beta_{1}, \beta_{2}, \beta_{3}\right)$, where each $\beta_{i} \in F_{i}$. The convention for labeling generators of the $F_{i}$ is explained in Figure 4 . 
Example 2 First consider the simplest nontrivial pure braids.

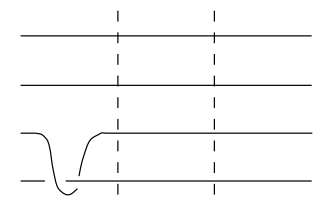

$$
\begin{aligned}
& \sigma_{1}{ }^{2}=\left(x_{1}, 1,1\right)=\left(1+X_{1}, 1,1\right) . \\
& {\sigma_{2}}^{2}=\left(1, x_{2}, 1\right)=\left(1,1+X_{2}, 1\right) . \\
& \sigma_{3}{ }^{2}=\left(1,1, x_{3}\right)=\left(1,1,1+X_{3}\right) .
\end{aligned}
$$

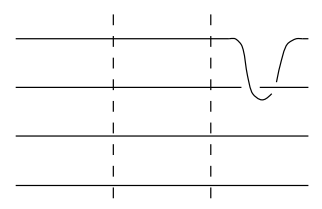

This shows that ${\sigma_{1}}^{2}>{\sigma_{2}}^{2}>{\sigma_{3}}^{2}>1$. In general, ${\sigma_{i}}^{2}>\sigma_{i+1}{ }^{2 N}$ for every power $N$, illustrating that for $n>2$, the Artin-Magnus ordering on $P_{n}$ is nonArchimedean (as it must be, by Hölder's theorem).

Example 3 The generator of the center of $P_{4}$ :
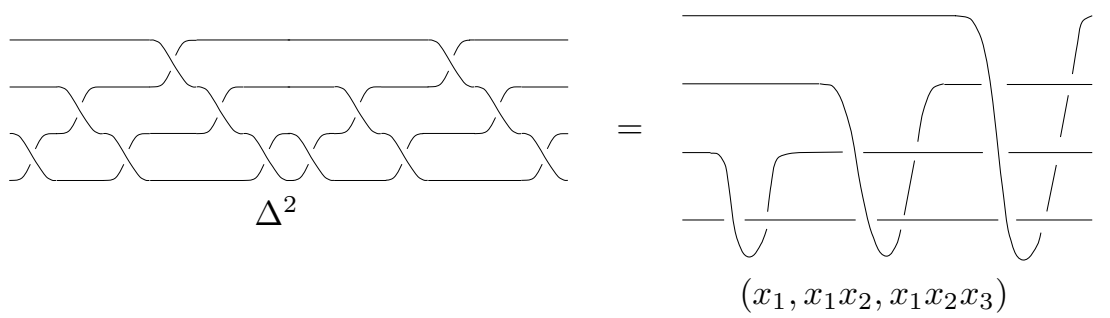

Example 4 The common braid used for hair, on the first three strings.

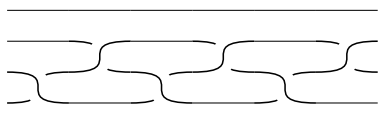

$\left(\sigma_{1} \sigma_{2}^{-1}\right)^{3}$

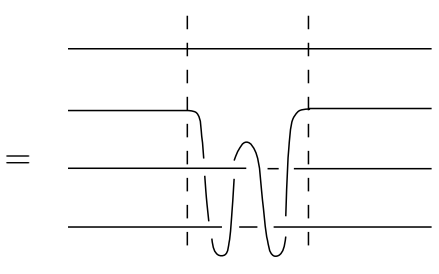

$\left(1, x_{2}{ }^{-1} x_{1}{ }^{-1} x_{2} x_{1}, 1\right)$ 
Applying the Magnus expansion to the second coordinate,

$$
\begin{aligned}
& x_{2}{ }^{-1} x_{1}{ }^{-1} x_{2} x_{1} \mapsto \\
& \quad\left(1-X_{2}+X_{2}{ }^{2}-X_{2}{ }^{3}+\cdots\right)\left(1-X_{1}+X_{1}{ }^{2}-X_{1}{ }^{3}+\cdots\right)\left(1+X_{2}\right)\left(1+X_{1}\right) \\
& \quad=1-X_{1} X_{2}+X_{2} X_{1}+\mathcal{O}(3)
\end{aligned}
$$

Since the first non-constant term is negative, we conclude $\left(\sigma_{1} \sigma_{2}{ }^{-1}\right)^{3}<1$.

The Artin-Magnus ordering is natural in several senses. Consider the increasing sequence

$$
P_{1} \subset P_{2} \subset \cdots \subset P_{n} \subset \cdots \subset P_{\infty},
$$

where the group $P_{\infty}$ is the direct limit.

Proposition 7 If $m<n$, the Artin-Magnus ordering of $P_{n}$, restricted to $P_{m}$, is the Artin-Magnus ordering of $P_{m}$. Therefore, this defines an ordering of $P_{\infty}$. Moreover, the retraction map $f: P_{n} \rightarrow P_{n-1}$ is as order-preserving as possible: $\beta<\gamma \Longrightarrow f(\beta) \leq f(\gamma)$.

Proof. The statement is obvious when considering coordinates: $P_{m}$ extends to $P_{n}$ by adding an appropriate number of "identity" coordinates. The retraction simply deletes the last coordinate.

It will be useful to consider the linking numbers associated with a pure braid $\beta$. Define the linking number of the $i$-th strand with the $j$-th strand, $i \neq j$ (indices refer to the left-endpoints), by

$$
\mathrm{lk}_{i, j}(\beta)=\frac{1}{2} \sum_{c} \operatorname{sign}(c),
$$

where the sum is over all crossings $c$ involving the two strings of index $i$ and $j$, and $\operatorname{sign}(c)$ is the power, \pm 1 , of the corresponding braid generator $\sigma_{k}{ }^{ \pm 1}$. Each $\mathrm{lk}_{i, j}: P_{n} \rightarrow \mathbf{Z}, 1 \leq i \neq j \leq n$ is additive: $\mathrm{lk}_{i, j}(\alpha \beta)=\mathrm{lk}_{i, j}(\alpha)+\mathrm{lk}_{i, j}(\beta)$.

Proposition 8 Let $\beta$ be a pure braid with $n$ strands, expressed in Artin coordinates: $\beta=\left(\beta_{1}, \ldots, \beta_{n-1}\right)$. Then the Magnus expansion

$$
\beta_{i}=1+q_{1} X_{1}+\cdots+q_{i} X_{i}+\mathcal{O}(2)
$$

in $F_{i} \subset \mathbf{Z}\left[\left[X_{1}, X_{2}, \ldots, X_{i}\right]\right]$ has coefficients of its linear terms:

$$
q_{j}=\mathrm{lk}_{j, i+1}(\beta) .
$$

Proof. The formula is a straightforward calculation, using two observations. First, the exponent sum of $x_{j}=x_{j, i}$ in a word $w=w\left(x_{1}, \ldots, x_{i}\right)$ equals the coefficient of $X_{j}$ in the Magnus expansion of $w$. Second, if $j \leq i$, then $x_{j, i}$ contributes +1 to $\mathrm{lk}_{j, i+1}(\beta)=\mathrm{lk}_{i+1, j}(\beta)$ and zero to all other linking numbers. 
The abelianization functor takes all the braid groups $P_{n}$ and $B_{n}, n \geq 2$, to infinite cyclic groups. The abelianization map $B_{n} \rightarrow \mathbf{Z}$ can be taken to be the total exponent count of a braid when expressed in the generators $\sigma_{i}$. This map sends $P_{n}$ to $2 \mathbf{Z}$; in this setting it can also be interpreted as twice the total linking number

$$
\beta \rightarrow 2 \sum_{1 \leq i<j \leq n} \mathrm{lk}_{i, j}(\beta) .
$$

The abelianization map on $P_{n}$ is clearly not order-preserving. However, it does respect the orderings if one confines attention to the free subgroups $F_{i} \subset P$ in the Artin decomposition

$$
P_{n}=F_{1} \ltimes F_{2} \ltimes F_{3} \ltimes \cdots \ltimes F_{n-1} .
$$

The abelianization of $F_{i}$ is the free abelian group of rank $i$, which we may identify with $i$-tuples of integers, $\mathbf{Z}^{i}$, and order lexicographically. In this setting the abelianization may be realized as the map $\operatorname{ab}_{i}(w)=\left(q_{1}, \ldots, q_{i}\right)$ where $q_{j}$ is the exponent sum of $x_{j}$ in $w$. We shall say that a mapping $x \rightarrow x^{\prime}$ of ordered sets is order-respecting if $x<y$ implies $x^{\prime} \leq y^{\prime}$. Then we see, from the definition of the Magnus ordering of $F_{i}$ that $\mathrm{ab}_{i}$ is order respecting. The next proposition follows directly from the above discussion.

Proposition 9 With the Artin-Magnus ordering on $P_{n}$ and lexicographic ordering on the product of infinite cyclic groups, the product of abelianization maps $\mathrm{ab}_{1} \times \mathrm{ab}_{2} \times \cdots \times \mathrm{ab}_{n-1}$, as a mapping

$$
P_{n}=F_{1} \ltimes F_{2} \ltimes \cdots \ltimes F_{n-1} \rightarrow \mathbf{Z} \times \mathbf{Z}^{2} \times \cdots \times \mathbf{Z}^{n-1},
$$

is order-respecting. Moreover, it is compatible with the retraction $f: P_{n} \rightarrow P_{n-1}$ in that the following diagram commutes:

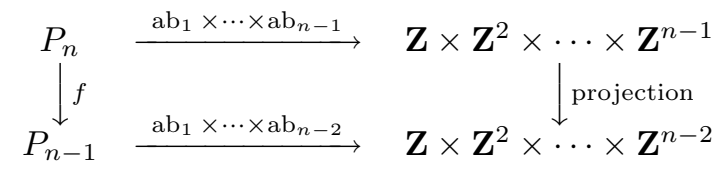

\section{Garside Positive Braids}

In his celebrated solution of the conjugacy problem in $B_{n}$, Garside [14] introduced the so-called "positive" braids, i.e., nontrivial braids which have an expression in the generators $\sigma_{i}$ without any negative exponents. We will call such braids "Garside positive." Let $B_{n}^{+}$denote the semigroup of Garside positive braids together with the identity.

Theorem 10 If a braid in $P_{n}$ is Garside positive, then it is also positive in the Artin-Magnus ordering. 
Proof. The statement is clear for elements of $P_{2}$. Inductively, suppose that it is true for $P_{n-1}$. Let $\beta \in P_{n}$ be Garside positive, and consider the braid $f(\beta) \in P_{n-1}$. Note that $f(\beta)$ is either Garside positive (case (1)) or has no crossings (case (2)). In case (1), applying induction, $f(\beta)$ is ArtinMagnus positive, hence $\beta$ is positive, by definition of the lexicographic order of $P_{n-1} \ltimes F_{n-1}$. In case (2), $\beta$ is an element of $F_{n-1}$. We may assume the first $n-1$ strings are straight and read the expression for $\beta$ in terms of the free generators of $F_{n-1}$ from the places where the $n^{\text {th }}$ string passes under the first $n-1$ strings. By our convention (choice of $x_{j, i}$ ), and Garside positivity, $\beta$ is therefore a strictly positive word in $x_{1}, \ldots, x_{n}$. It follows from Proposition 8 that its Magnus expansion has positive leading coefficient (occurring at a linear term). Therefore $\beta>1$.

Recall that an ordered set is said to be well-ordered if every non-empty subset has a least element.

Theorem 11 The set of Garside positive pure n-braids is well-ordered by the Artin-Magnus ordering.

Proof. The statement is clear for $n=2$. Now suppose that $n>2$ and the theorem holds for $n-1$. Consider a non-empty set $S \subset P_{n}$ of Garside positive braids. We need to show $S$ has a least element. The set $f(S)=\left\{f(\beta) \in P_{n-1} \mid\right.$ $\beta \in S\}$, being a subset of $P_{n-1} \cap B_{n-1}^{+}$, has a least element; call it $\alpha_{0}$. Let

$$
S_{0}=\left\{\gamma \in S \mid f(\gamma)=\alpha_{0}\right\}
$$

clearly if there is a least element of $S_{0}$ it is also the least element of $S$. Note that the coordinates of $\gamma \in S_{0}$ in $P_{n-1} \ltimes F_{n-1}$ are $\gamma=\left(\alpha_{0}, \alpha_{0}{ }^{-1} \gamma\right)$. We now appeal to lemma 12 to find a $\gamma_{0} \in S_{0}$ whose last coordinate $\alpha_{0}{ }^{-1} \gamma_{0}$ is minimal. Then $\gamma_{0}$ is the least element of $S$.

Lemma 12 Consider a subset $T \subset F_{n-1} \subset P_{n}$ of the form

$$
T=\left\{\alpha_{0}{ }^{-1} \gamma \mid \gamma \in S_{0}\right\},
$$

where $S_{0}$ is some set of Garside-positive pure $n$-braids and $\alpha_{0}$ is a fixed pure braid in $P_{n-1} \subset P_{n}$. Then $T$ has a least element in the Magnus ordering of $F_{n-1}$.

Proof. The condition $\alpha_{0}^{-1} \gamma \in F_{n-1}$ is equivalent to the equation $f(\gamma)=$ $\alpha_{0}$. Since $\alpha_{0}$ is in $P_{n-1}$, and $\gamma$ is Garside positive, we have $\operatorname{lk}_{j, n}\left(\alpha_{0}{ }^{-1} \gamma\right)=$ $\mathrm{lk}_{j, n}(\gamma) \geq 0$. This implies that the coefficients $\left(q_{1}, \ldots, q_{n-1}\right)$ of the linear terms in the Magnus expansion of all elements of $T$ are positive, hence there is a lexicographically minimal one, say $\left(\hat{q}_{1}, \ldots, \hat{q}_{n-1}\right)$. Let

$$
T^{\prime}=\left\{\tau \in T \mid \tau=1+\hat{q}_{1} X_{1}+\cdots \hat{q}_{n-1} X_{n-1}+\mathcal{O}(2)\right\} .
$$


Then $T^{\prime}$ is nonempty, and its elements are $<$ other elements of $T$. We now claim that $T^{\prime}$ is finite. It follows that $T^{\prime}$ has a least element, which is also the least element of $T$. To verify the claim, observe that the exponent sum (as a word in $\left.x_{1}, \ldots, x_{n-1}\right)$ of every element of $T^{\prime}$ is $\hat{q}_{1}+\cdots+\hat{q}_{n-1}$. Each $x_{i}$ has exponent sum +2 when expanded in the braid generators $\sigma_{j}$. The $\sigma$-exponent sum is an invariant of braids, and we use it to conclude that for every $\alpha_{0}{ }^{-1} \gamma \in T^{\prime}$, the length of $\gamma$ is exactly $2 \sum_{i} \hat{q}_{i}$ minus the exponent sum of $\alpha_{0}$. There are only finitely many distinct $\gamma$ satisfying this.

We note that the analogue of Theorem 11 was proved in $[6,18]$ for the Dehornoy ordering of the full braid groups.

We next compare the Artin-Magnus ordering $<_{A M}$ on $P_{n}$ with Dehornoy's and several other orderings on the braid groups appearing in the literature.

\section{The Garside partial order}

Garside [14] defined a partial order $\prec$ on the semigroup $B_{n}^{+}$by $\alpha \prec \beta$ for $\alpha, \beta \in B_{n}^{+}$, if there exists a $\gamma \in B_{n}^{+}$such that $\alpha \gamma=\beta$. Thurston [11] showed that this in fact defines a lattice order on the set of non-repeating braids $D=$ $\left\{\alpha \in B_{n}^{+} \mid \alpha \prec \Delta\right\}$.

Proposition 13 The Artin-Magnus order on $P_{n}$ extends the Garside order on $P_{n}^{+}=P_{n} \cap B_{n}^{+}$.

Proof. This follows immediately from Theorem 10.

\section{The partial order of Elrifai and Morton}

In [10], Elrifai and Morton define a partial order on $B_{n}$, as follows: for $\alpha, \beta \in B_{n}$, write $\alpha \leq_{E M} \beta$ when $\beta=\gamma_{1} \alpha \gamma_{2}$, for some $\gamma_{1}, \gamma_{2} \in B_{n}^{+}$. Then $1 \leq_{E M} \alpha$ if and only if $\alpha \in B_{n}^{+}$. Furthermore, taking $\Delta=\Delta_{n} \in B_{n}$ to be the "half-twist" braid, defined inductively by $\Delta_{2}=\sigma_{1}, \Delta_{n}=\Delta_{n-1} \sigma_{n-1} \cdots \sigma_{1}$, they show that in their partial order each generator $\sigma_{i}$ satisfies

$$
1 \leq_{E M} \sigma_{i} \leq_{E M} \Delta
$$

We remark that $1 \leq_{E M} \sigma_{i}{ }^{2} \leq_{E M} \Delta^{2}$ holds also for each generator $\sigma_{i}$, since $\Delta=\gamma_{1} \sigma_{i}=\sigma_{i} \gamma_{2}$, for some $\gamma_{1}, \gamma_{2} \in B_{n}^{+}$. Hence $\Delta^{2}=\gamma_{1} \sigma_{i}{ }^{2} \gamma_{2}$. This is consistent with the Artin-Magnus order on $P_{n}$ :

Proposition 14 For each generator $\sigma_{i} \in B_{n}$,

$$
1<_{A M} \sigma_{i}^{2} \leq_{A M} \Delta^{2}
$$

We have equality in the second inequality only when $i=1, n=2$. 
Proof. The inequalities follow immediately from example 3.

However, the following example shows that the Artin-Magnus order on $P_{n}$ does not extend the partial order defined by Elrifai and Morton. Let $\alpha=$ $\sigma_{1}^{2} \sigma_{2}{ }^{-2}$. This has Artin-combing $\left(x_{1}, x_{2}{ }^{-1}\right)$, hence is positive in the ArtinMagnus ordering. Taking $\gamma_{1}=\sigma_{1} \sigma_{2}$ and $\gamma_{2}=\sigma_{2} \sigma_{1}$, and defining $\beta=\gamma_{1} \alpha \gamma_{2}$, we have $\beta<_{A M} 1$, so in particular, $\beta<\alpha$ in the Artin-Magnus order. We note that $\gamma_{1} \alpha \gamma_{1}{ }^{-1}$ is also negative in the Artin-Magnus ordering - conjugation by non-pure braids is not order-preserving.

\section{The Dehornoy ordering}

The Dehornoy ordering [8] on $B_{n}$ may be defined in terms of the generators $\sigma_{1}, \cdots, \sigma_{n-1}$ as follows: A braid $\beta$ is in the positive cone if and only if there is, for some $i \in\{1, \ldots, n-1\}$, an expression

$$
\beta=w_{1} \sigma_{i} w_{2} \sigma_{i} \cdots w_{k-1} \sigma_{i} w_{k}
$$

in which each $w_{j}$ is a word in $\sigma_{i+1}^{ \pm 1}, \cdots, \sigma_{n-1}^{ \pm 1}$. In other words, the generator with the lowest subscript appears with only positive exponent. A more geometric view of the same ordering appears in [13]. Then we define a right-ordering by $\alpha<\gamma$ iff $\gamma \alpha^{-1}$ is in the positive cone. (Actually Dehornoy chose his ordering to be left-invariant by using the criterion $\alpha^{-1} \gamma$ positive, but the choice is arbitrary and right-invariance seems to dominate the literature on ordered groups.)

As noted already, no ordering of $B_{n}$ can be a bi-ordering, for $n \geq 3$. Even when restricted to $P_{n}$, as noted in [13], the Dehornoy ordering is not bi-invariant. However, there are some similarities between the Dehornoy and Artin-Magnus orderings. In both orderings we have

$$
\sigma_{1}^{2} \gg{\sigma_{2}}^{2} \gg{\sigma_{3}}^{2} \gg \cdots>1,
$$

where the notation $\alpha \gg \beta$ means that $\alpha$ is greater than all powers of $\beta$. Another similarity is that the Dehornoy ordering is also a well-ordering when applied to Garside positive braids (see [18] and [6]).

On the other hand, the pure braid $\left(\sigma_{1} \sigma_{2}{ }^{-1}\right)^{3}$ is clearly Dehornoy positive, whereas we saw that it is negative in the Artin-Magnus ordering. Of course, its inverse is Dehornoy negative and Artin-Magnus positive.

We wish to thank R. Fenn for pointing out the following proposition. It shows that the Dehornoy ordering is fundamentally different from our ordering of $P_{n}$.

Theorem $15 B_{n}$ has a least positive element in the Dehornoy ordering, namely $\sigma_{n-1}$. Similarly, $\sigma_{n-1}{ }^{-1}$ is the greatest element which is $<1$ in $B_{n}$. In $P_{n}$, $\sigma_{n-1}^{2}$ is the least Dehornoy-positive, and $\sigma_{n-1}{ }^{-2}$ is the greatest element which is $<1$ in the Dehornoy ordering. 
Proof. If a braid has the form of eq. (4), call it $i$-positive, so that $\beta \in B_{n}$ is Dehornoy-positive if and only if it is $i$-positive for some $i=1, \ldots, n-1$. To prove the first statement, note that $\sigma_{n-1}$ is $(n-1)$-positive. Suppose that there is a $\beta \in B_{n}$ with $1<\beta<\sigma_{n-1}$. Then $\beta \sigma_{n-1}{ }^{-1}<1$ by right-invariance. Now $\beta$ is $i$-positive for some $i$. If $i<n-1$ we conclude $\beta \sigma_{n-1}{ }^{-1}$ is also $i$ positive, contradicting $\beta \sigma_{n-1}{ }^{-1}<1$. On the other hand, if $i=n-1, \beta$ must be a positive power of $\sigma_{n-1}$, contradicting $\beta<\sigma_{n-1}$. This establishes the first statement. The other parts follow similarly.

Corollary 16 The Dehornoy ordering of $B_{n}$ is discrete: every element $\beta$ has a unique successor, $\sigma_{n-1} \beta$, and predecessor, $\sigma_{n-1}{ }^{-1} \beta$. Similarly, in the Dehornoy ordering restricted to $P_{n}$, a pure braid $\beta$ is the only element of $P_{n}$ strictly between $\sigma_{n-1}{ }^{-2} \beta$ and $\sigma_{n-1}{ }^{2} \beta$.

Theorem 17 For $n \geq 3$, the Artin-Magnus ordering of $P_{n}$ is order-dense: given any two pure $n$-braids $\alpha<\gamma$, there exist (infinitely many) pure braids $\beta$ with $\alpha<\beta<\gamma$.

Proof. By invariance, we may assume $\alpha=1$. In the free group $F_{n-1}=$ $\left\langle x_{1}, \ldots, x_{n-1}\right\rangle$, consider the sequence of commutators $\left\{c_{i}\right\}$ defined recursively by

$$
c_{1}=x_{1} x_{2} x_{1}^{-1} x_{2}{ }^{-1}, \quad c_{k}=x_{1} c_{k-1} x_{1}{ }^{-1} c_{k-1}{ }^{-1} .
$$

A simple calculation shows that their Magnus expansions are, in ascending order:

$$
c_{k}=1+X_{1}{ }^{k} X_{2}-k X_{1}{ }^{k-1} X_{2} X_{1}+\cdots .
$$

It follows that each $c_{k}>1$ in the Magnus order of $F_{n-1}$, but that $c_{k} \rightarrow 1$ in the sense that if $1<w \in F_{n-1}$, then $1<c_{k}<w$ for sufficiently large $k$; just take $k$ bigger than the degree of the first nonzero non-constant term of the Magnus expansion of $w$. Now, in $P_{n}$, consider the sequence of braids $\{\beta(k)\}$ with Artin coordinates

$$
\beta(k)=\left(1, \ldots, 1, c_{k}\right) .
$$

It is clear that given any $\gamma>1$ in $P_{n}$, we have $1<\beta(k)<\gamma$ for all sufficiently large $k$.

Since for $n \geq 3, P_{n}$ with the Artin-Magnus ordering is a countable totally ordered set, dense and with no greatest or least element, it is order-isomorphic with the rational numbers, by a classical result. Of course this order-isomorphism cannot be an algebraic isomorphism, as $P_{n}$ is non-abelian.

We note that for $B_{\infty}$ or $P_{\infty}$ the Dehornoy ordering is no longer discrete, but is (like the Artin-Magnus ordering) order-dense.

Theorem 18 For $n \geq 3$, the Artin-Magnus ordering on $P_{n}$ does not extend to any right-ordering on $B_{n}$. It also does not extend to a left-invariant ordering on $B_{n}$. 
Proof. We may assume that $n=3$. It has already been shown that $\left(\sigma_{1} \sigma_{2}{ }^{-1}\right)^{3}<1$. A similar calculation shows that $\left(\sigma_{1}{ }^{-1} \sigma_{2}\right)^{3}<1$. If the ordering extends to a right- (or left-) invariant ordering of $B_{3}$, we must have $\sigma_{1} \sigma_{2}{ }^{-1}<1$ and $\sigma_{1}{ }^{-1} \sigma_{2}<1$.

Assuming right invariance we conclude

$$
\sigma_{1} \sigma_{2}{ }^{-1} \sigma_{1}{ }^{-1} \sigma_{2}<\sigma_{1}^{-1} \sigma_{2}<1,
$$

which would imply $\left(\sigma_{1} \sigma_{2}{ }^{-1} \sigma_{1}{ }^{-1} \sigma_{2}\right)^{3}<1$. But a direct calculation shows

$$
\left(\sigma_{1} \sigma_{2}{ }^{-1} \sigma_{1}{ }^{-1} \sigma_{2}\right)^{3}=\left(1, x_{2}{ }^{-1} x_{1}{ }^{-1} x_{2}{ }^{-1} x_{1} x_{2}{ }^{2}\right)=\left(1,1+X_{1} X_{2}+\cdots\right)>1,
$$

a contradiction showing no right-invariant extension to $B_{3}$ exists.

If instead we assume left-invariance, we argue that

$$
\sigma_{1} \sigma_{2}{ }^{-1} \sigma_{1}{ }^{-1} \sigma_{2}<\sigma_{1} \sigma_{2}^{-1}<1,
$$

and reach the same contradiction.

Recently, Rhemtulla and Rolfsen [27] have shown that no right- or leftinvariant ordering of $B_{n}$ whatsoever can be bi-invariant when restricted to $P_{n}$, or any finite index subgroup.

\section{Fibre-type hyperplane arrangements}

A hyperplane arrangement is a collection of complex $(n-1)$-dimensional hyperplanes in complex $n$-space. The theory of hyperplane arrangements is a deep subject with many unsolved problems. The fundamental group of the complement of the union of the hyperplanes is an important invariant of an arrangement (see the recent survey paper by Paris [25]). It is conjectured, but not known in general, that all such groups are torsion-free.

The pure braid group $P_{n}$ can be viewed as the fundamental group of the complement of the hyperplanes $z_{i}=z_{j}, 1 \leq i<j \leq n$ in $\mathbf{C}^{n}$. Fibre-type hyperplane arrangements are defined in [12], and are in a sense a generalization of this example.

Theorem 19 Let $G$ be the fundamental group of (the complement of) a fibretype hyperplane arrangement. Then $G$ is bi-orderable.

Proof. By definition of a fibre-type arrangement, its complement $M_{r}$ is the top of a tower of fibrations $M_{i} \rightarrow M_{i-1}$, with fibre the complex plane minus $d_{i}$ points. The space $M_{1}$ at the bottom of the tower is likewise a punctured complex plane. Therefore $\pi_{1}\left(M_{i}\right) \cong \pi_{1}\left(M_{i-1}\right) \ltimes F_{d_{i}}$. Moreover, according to [12], proposition 2.5, the action of $\pi_{1}\left(M_{i-1}\right)$ upon $F_{d_{i}}$ is trivial on $H_{1}\left(F_{d_{i}}\right)$. It follows, in the same way as Theorem 6 , that if each free group $F_{d_{i}}$ is given the Magnus ordering, then the lexicographic ordering on

$$
\left.G \cong\left(\cdots\left(F_{d_{1}} \ltimes F_{d_{2}}\right) \ltimes F_{d_{3}}\right) \ltimes \cdots \ltimes F_{d_{r}}\right)
$$

is bi-invariant. 


\section{References}

[1] Emil Artin. Theorie der Zöpfe. Abh. Math. Sem. Univ. Hamburg, 4:47-72, 1925.

[2] Emil Artin. Theory of braids. Ann. of Math., 48:101-126, 1947.

[3] George Bergman. Ordering co-products of groups. Journal of Algebra, 133:313-339, 1990.

[4] G. Birkhoff. Review of Everett and Ulam, On ordered groups. Mathematical Reviews MR 7.4g

[5] Joan S. Birman. Braids, Links and Mapping Class Groups. Number 82 in Annals of Mathematics Studies. Princeton University Press, Princeton, 1975 .

[6] Serge Burckel. The well-ordering on positive braids. J. Pure Appl. Algebra, 120(1):1-17, 1997.

[7] Gerhard Burde and Heiner Zieschang. Knots. de Grutyer, Inc., New York, 1989.

[8] Patrick Dehornoy. From large cardinals to braids via distributive algebra. J. Knot Theory Ramifications, 4(1):33-79, 1995.

[9] C. J. Everett and S. Ulam. On ordered groups. Trans. Amer. Math. Soc. 57, 208-216, 1945.

[10] Elsayed A. Elrifai and H. R. Morton. Algorithms for positive braids. Quart. J. Math. Oxford Ser. (2), 45(180):479-497, 1994.

[11] David B. A. Epstein, James W. Cannon, Derek F. Holt, Silvio V. F. Levy, Michael S. Paterson, and William P. Thurston. Word processing in groups. Jones and Bartlett Publishers, Boston, MA, 1992.

[12] Michael Falk and Richard Randell. The lower central series of a fiber-type arrangement. Invent. Math., 82(1):77-88, 1985.

[13] Roger Fenn, Michael Greene, Dale Rolfsen, Colin Rourke, and Bert Wiest. Ordering the braid groups. To appear, Pacific J. Math., 1998.

[14] F.A. Garside. The braid group and other groups. Quart. J. Math., 20:235254, 1969.

[15] O. Hölder. Die axiome der Quantität und die Lehre von Mass. Math.-Phys. Kl., 53:1-64, 1901.

[16] Kenkichi Iwasawa. On linearly ordered groups. Journal for the Mathematical Society of Japan, 1(1):1-9, 1948. 
[17] A. I. Kokorin and V. M. Kopytov. Fully ordered groups, volume 820 of Lecture Notes in Mathematics. Wiley and Sons, New York, 1974.

[18] Richard Laver. Braid group actions on left distributive structures, and well orderings in the braid groups. J. Pure Appl. Algebra, 108(1):81-98, 1996.

[19] A. I. Mal'cev. On the embedding of group algebras in division algebras Dokl. Akad. Nauk SSSR60, 1499-1501, 1948.

[20] Wilhelm Magnus, Abraham Karrass, and Donald Solitar. Combinatorial group theory. Dover Publications Inc., New York, revised edition, 1976. Presentations of groups in terms of generators and relations.

[21] Roberta Mura and Akbar Rhemtulla. Orderable Groups, volume 27 of Lecture Notes in Pure and Applied Mathematics. Marcel Dekker, New York, 1977.

[22] B. H. Neumann. On ordered division rings Trans. Amer. Math. Soc. 66:202-252, 1949.

[23] B. H Neumann. On ordered groups Amer. J. Math, 71, 1-18, 1949.

[24] L. P. Neuwirth. The status of some problems related to knot groups. In Topology Conference (Virginia Polytech. Inst. and State Univ., Blacksburg, Va., 1973), pages 209-230. Lecture Notes in Math., Vol. 375. Springer, Berlin, 1974.

[25] Luis Paris. On the fundamental group of the complement of a complex hyperplane arrangement. To Appear, 1998.

[26] Donald S. Passman. The Algebraic Structure of Group Rings. WileyInterscience Press, New York, 1977.

[27] Akbar Rhemtulla and Dale Rolfsen. Local indicability in ordered groups: barids and elementary amenable groups Proc. Amer. Math. Soc. to appear.

[28] Dale Rolfsen and Jun Zhu. Braids, orderings and zero divisors J. Knot Theory and Ramifications7, 837-841, 1998.

[29] Hamish Short and Bert Wiest. Orderings of mapping class groups after Thurston. Enseign. Math.46, 279-312, 2000.

[30] A.A. Vinogradov. On the free product of ordered groups. Mat. Sb., 25:163$168,1949$. 